\title{
Macrophages inhibit adipogenic differentiation of adipose tissue derived mesenchymal stem/stromal cells by producing pro-inflammatory cytokines
}

\author{
Hui Ma ${ }^{1 \dagger}$, Ya-nan $\mathrm{Li}^{1 \dagger}$, Lin Song ${ }^{1}$, Rui Liu ${ }^{1}$, Xiaolei Li ${ }^{1}$, Qianwen Shang ${ }^{1}$, Ying Wang ${ }^{2}$, Changshun Shao ${ }^{3 *}$ (D)
} and Yufang Shi ${ }^{1 *}$

\begin{abstract}
Background: Mesenchymal stem/stromal cells (MSCs) and macrophages are critical components in many tissue microenvironments, including that in adipose tissue. The close interaction between MSCs and macrophages modulates various adipose-related disease development. However, the effects of macrophages on the fate of MSCs remain largely elusive. We here studied the effect of macrophages on the adipogenic differentiation of MSCs.

Methods: Macrophages were obtained from THP-1 cells treated with phorbol-12-myristate-13-acetate (PMA). The induced matured macrophages were then induced to undergo classically activated macrophage (M1) or alternatively activated macrophage (M2) polarization with lipopolysaccharide (LPS)/interferon (IFN)- $\gamma$ and interleukin (IL)-4/IL-13, respectively. The supernatants derived from macrophages under different conditions were applied to cultured human adipose tissue-derived mesenchymal stem/stromal cells (hADSCs) undergoing adipogenic differentiation. Adipogenic differentiation was evaluated by examining Oil Red $\mathrm{O}$ staining of lipid droplets and the expression of adipogenesisrelated genes with real-time quantitative polymerase chain reaction (Q-PCR) and western blot analysis.

Results: The adipogenic differentiation of hADSCs was impaired when treated with macrophage-derived supernatants, especially that from the M1-polarized macrophage (M1-sup). The inhibitory effect was found to be mediated by the inflammatory cytokines, mainly tumor necrosis factor- $\alpha$ (TNF- $\alpha$ ) and IL-1 $\beta$. Blocking TNF- $\alpha$ and IL-1 $\beta$ with neutralizing antibodies partially alleviated the inhibitory effect of M1-sup.

Conclusion: Macrophage-derived supernatants inhibited the adipogenic differentiation of hADSCs in vitro, irrespective of the polarization status (M0, M1 or M2 macrophages). M1-sup was more potent because of the higher expression of pro-inflammatory cytokines. Our findings shed new light on the interaction between hADSCs
\end{abstract}

\footnotetext{
*Correspondence: shaoc@suda.edu.cn; yfshi@suda.edu.cn

${ }^{\dagger}$ Hui Ma and Ya-nan Li contributed equally to this work

${ }^{1}$ The First Affiliated Hospital of Soochow University, Institutes

for Translational Medicine, State Key Laboratory of Radiation Medicine and Protection, Key Laboratory of Stem Cells and Medical Biomaterials of Jiangsu Province, Medical College of Soochow University,

Suzhou 215123, Jiangsu, China

${ }^{3}$ Institutes for Translational Medicine, State Key Laboratory of Radiation

Medicine and Protection, Soochow University, Suzhou 215123, Jiangsu,

China

Full list of author information is available at the end of the article
}

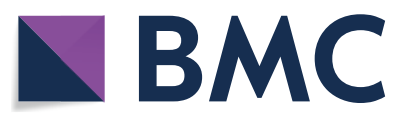

(c) The Author(s) 2020. This article is licensed under a Creative Commons Attribution 4.0 International License, which permits use, sharing, adaptation, distribution and reproduction in any medium or format, as long as you give appropriate credit to the original author(s) and the source, provide a link to the Creative Commons licence, and indicate if changes were made. The images or other third party material in this article are included in the article's Creative Commons licence, unless indicated otherwise in a credit line to the material. If material is not included in the article's Creative Commons licence and your intended use is not permitted by statutory regulation or exceeds the permitted use, you will need to obtain permission directly from the copyright holder. To view a copy of this licence, visit http://creativeco mmons.org/licenses/by/4.0/. The Creative Commons Public Domain Dedication waiver (http://creativecommons.org/publicdomain/ zero/1.0/) applies to the data made available in this article, unless otherwise stated in a credit line to the data. 
and macrophages and have implications in our understanding of disrupted adipose tissue homeostasis under inflammation.

Keywords: Macrophages, hADSCs, Adipogenesis, Inflammation cytokines

\section{Background}

Mesenchymal stem/stromal cells (MSCs), a heterogeneous stem cell population, were first found in bone marrow by Friedenstein et al. [1] and were subsequently isolated from various other tissues, such as adipose tissue, umbilical cord and dental pulp [2,3]. MSCs are now characterized by their abilities to self-renew and give rise to multiple lineages including osteoblasts, chondrocytes and adipocytes [4-6]. As a major source of adipocytes, MSCs first differentiate into preadipocytes that are committed to the adipogenic lineage. Preadipocytes then give rise to enlarged mature adipocytes that can synthesize lipid droplets, secrete specific adipocyte factors and regulate energy metabolism [7]. Adipocyte differentiation from MSCs is believed to play a vital role in maintaining the adipose tissue homeostasis [8]. Recently, emerging evidence has demonstrated that MSCs interact with both innate and adaptive immune systems to modulate local immune response [9]. IFN- $\gamma$ in combination with any one of TNF- $\alpha / \mathrm{IL}-1 \alpha / \mathrm{IL}-1 \beta$ can endow MSCs with immunomodulatory capability, mainly in an indoleamine 2, 3-dioxidase (IDO)/inducible nitric oxide synthase (iNOS)-dependent manner hinging on species difference $[10,11]$. Accordingly, MSCs-based cell therapy can modulate immune microenvironment and dampen immune and inflammatory responses associated with graft-versus-host-disease (GVHD) [12], systemic lupus erythematosus (SLE) [13] and experimental autoimmune encephalomyelitis (EAE) [14].

Macrophages, a critical component in tissue microenvironment, contribute to the maintenance or disruption of homeostasis via the functionally distinct subpopulations in response to different microenvironmental cues [15]. There are two main types of activation and polarization states in mammals: M1 and M2 [16, 17]. The imbalance between M1 and M2 macrophages has been found to be responsible for chronic inflammatory milieu in adipose tissue and insulin sensitivity [18]. In lean individuals, macrophages dispersed throughout adipose tissues are predominantly resident macrophages and are polarized toward M2 phenotype, while in obese individuals there are an elevated number of infiltrating macrophages of activated pro-inflammatory phenotype, namely M1 subtype, in adipose tissue [19]. Interestingly, macrophages are remarkably plastic, the polarized M1/M2 phenotype can, to some extent, be experimentally reversed in vitro and in vivo, which makes macrophages as effect target for immunomodulatory therapeutic applications [20]. Local cytokines milieu, reactive oxygen species (ROS) and metabolism pathway can all direct macrophage polarization $[21,22]$.

Adipose tissues are responsible for storing energy and consist of a large number of clusters of fat cells and immune cells, such as macrophages, as mentioned above [23]. Macrophages play an indispensable role in maintaining adipose-tissue homeostasis. Zheng et al. demonstrated that macrophage accumulation in adipose tissue during obesity is initiated by in situ proliferation of resident adipose tissue macrophages (ATMs) and further augmented by monocyte migration and subsequent macrophage differentiation in the late stage [24]. Furthermore, CD11b (integrin $\alpha_{\mathrm{M}}$ ) deficiency resulted in impaired monocyte migration and improved insulin resistance (IR) [25]. However, the interaction between adipocyte precursor, namely MSCs, and macrophages in adipose tissue in situ is still elusive. Recently, the complex cross-talk between MSCs and macrophages has attracted significant attention. MSCs respond to macrophages and then affect their polarization by secreting factors or other effector [26], such as IL-6 [27], prostaglandin E2 (PGE2) [28] and exosome [29]. Extensive studies have shown that macrophages could also promote the proliferation of MSCs and possessed the capability to enhance osteogenesis of MSCs in an oncostain M (OSM) signaling pathway dependent manner [30], indicating a critical interaction between MSCs and macrophages under the condition of physiological response during bone injury and bone remodeling.

Here, we studied the effect of macrophages on the adipogenic differentiation of human hADSCs in vitro. We observed a reduced adipogenic differentiation in hADSCs treated with macrophage-derived culture supernatants, especially that from M1 macrophage. Furthermore, inflammatory cytokines TNF- $\alpha$ and IL- $1 \beta$ secreted by M1 macrophage were found to account for the inhibitory effect. These findings expand our understanding of cellular interaction between hADSCs and macrophages.

\section{Results}

\section{Characterization of polarized macrophage}

Human THP-1 monocyte cells were treated with PMA to generate macrophages (Additional file 1: Fig. S1a), as previously reported [31]. Mature macrophages were confirmed by flow cytometry to express CD11b upon 
PMA treatment (M0 macrophage) compared with control THP-1 (Additional file 1: Fig. S1b). M0 macrophages were then polarized toward M1 and M2 with LPS/IFN- $\gamma$ and IL-4/IL-13, respectively. M1-polarized macrophage exhibited significantly higher expressions of IL-1 $\beta$, IL-12, IL-6 and TNF- $\alpha$ than the M0 and M2 macrophages (Additional file 1: Fig. S1c-f). In contrast, M2 macrophages were shown to highly express $\mathrm{CC}$ chemokine ligand 22 (CCL22), transglutaminase 2 (TGM2) and peroxisome proliferator-activated receptor $\gamma($ PPAR- $\gamma)$ (Additional file 1: Fig. S1g-i).

\section{Macrophage-derived supernatants inhibit adipocyte differentiation of hADSCs}

hADSCs at 3th passage were identified by their expression of MSC surface markers through flow cytometry assay. These cells were positive for CD73, CD105, CD90, CD29 but negative for CD11b, CD31, CD34, CD45 (Additional file 1: Fig. S2a). Furthermore, we cultured hADSCs in differentiation medium and examined their adipogenic and osteogenic potentials by Oil Red O or Alizarin Red $\mathrm{S}$ staining, respectively (Additional file 1: Fig. S2b). They could be induced to exhibit enormous lipid droplets and increased calcium accumulation, respectively.

To ascertain the effect of macrophages on adipogenic differentiation of hADSCs in vitro, M1 and M2 macrophages were prepared and fresh culture supernatants were respectively collected $24 \mathrm{~h}$ after removal of stimuli. The adipogenic differentiation of hADSCs was evaluated in the presence of different macrophage supernatants (M0-sup, M1-sup or M2-sup) (Fig. 1a). After 9-day differentiation, a remarkably repressed formation of lipid droplets was observed in the hADSCs treated with macrophage supernatants, especially M1-sup (Fig. 1b). Consistently, a dramatic decrease in the expression of adipogenic differentiation related genes was observed, such as PPAR- $\gamma$, glucose transporter 4 (Glut4), CCAAT/ enhancer binding proteins $\beta(\mathrm{C} / \mathrm{EBP}-\beta), \mathrm{CCAAT} /$ enhancer binding proteins $\gamma(\mathrm{C} / \mathrm{EBP}-\gamma)($ Fig. $1 \mathrm{c}, \mathrm{d})$. Interestingly, we found that pre-treatment of macrophageconditioned supernatants also endowed hADSCs resistance to adipogenic differentiation (Fig. 1e), indicating that the inhibitory effect did not require the continuous presence of the supernatant. Taken together, these results suggested that the macrophage supernatants, irrespective of the polarization status of the macrophages, impaired the adipogenic differentiation potential of hADSCs.

\section{The inhibitory effect of macrophage culture supernatants is dependent on secretory proteins}

The components in macrophage supernatants may include cytokines, neutral proteases, chemokines, complement components, arachidonic acid metabolites and lactic acid. To further determine the components which are responsible for the inhibitory effect on adipogenesis of hADSCs, ingredients were divided into two fractions, one with components $>3 \mathrm{kDa}$ and one with $<3 \mathrm{kDa}$. We found that only the ingredients in the $>3 \mathrm{kDa}$, but not those in the $<3 \mathrm{kDa}$, could inhibit the formation of lipid droplets, as shown by Oil Red $\mathrm{O}$ staining (Fig. 2a). Accordingly, we found decreased expression of PPAR- $\gamma, \mathrm{C} / \mathrm{EBP}-\alpha$ and $\mathrm{C} / \mathrm{EBP}-\beta$ in hAD$\mathrm{SCs}$ treated with $>3 \mathrm{kDa}$ fraction (Fig. $2 \mathrm{~b}, \mathrm{c}$ ). In addition, the M1-sup was more potent than M0/M2-sup in inhibiting adipocyte differentiation. These data indicate that large molecules, rather than small molecules or metabolites, produced by macrophages were responsible for the inhibitory effect on adipogenic differentiation of hADSCs.

\section{Pro-inflammatory cytokines TNF- $\alpha$ and IL-1 $\beta$ inhibit adipocyte differentiation of hADSCs}

The accumulation of adipose tissue macrophages (ATMs) is a significant characteristic of obesity-associated chronic inflammation, the ATMs are also critical in regulating obesity development. Macrophages form the so-called 'crown-like structures'(CLSs) around adipocytes during obesity, and mainly exhibit M1 phenotype $[19,32]$. Considering that the M1 macrophages produce various types of inflammatory cytokines, such as TNF- $\alpha$ and IL-1 $\beta$, which play an important role in the propagation of obesity-related inflammation [33], we thus focused on the pro-inflammatory cytokines in M1-sup. TNF- $\alpha$, IL-6, IL-12 and IL-1 $\beta$, alone or in combination, were added to hADSCs during the induction of adipogenic differentiation. We found that the adipocyte differentiation of hADSCs was significantly inhibited by TNF- $\alpha$ or IL- $1 \beta$ treatment in a concentration-dependent manner, as shown by Oil Red O staining and Q-PCR assay (Fig. 3a, Additional file 1: Fig. S3a-c). However, the adipogenic differentiation of hADSCs was not altered by IL- 6 and IL-12, when they were used alone or in combination (Fig. 3a). Next, Q-PCR assay showed that key adipogenesis related genes, including PPAR- $\gamma$, C/EBP- $\alpha$, Glut4 and lipoprotein lipase (LPL), were also evidently decreased by TNF- $\alpha$ or IL- $1 \beta$ at a very lower concentration during adipogenic differentiation (Fig. 3b). Furthermore, Western blot assay further confirmed that TNF- $\alpha$ and IL- $1 \beta$ inhibited the expression of PPAR- $\gamma$, a master transcription factor for adipogenesis, in differentiated hADSCs (Fig. 3c). Thus, TNF- $\alpha$ and IL- $1 \beta$, cytokines that are highly expressed in M1 polarized macrophage, can potently inhibit the adipocyte differentiation of hADSCs. 
a

$A D: M \varphi$ supernatant $=1: 1$

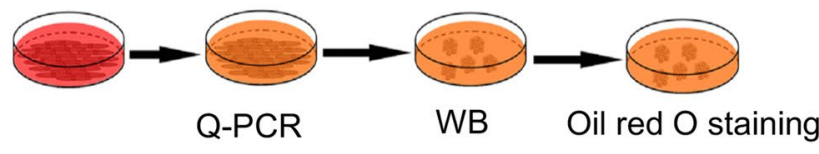

Q-PCR WB Oil red $O$ staining

Day $0 \quad$ Day $3 \quad$ Day 5 Day 9
C

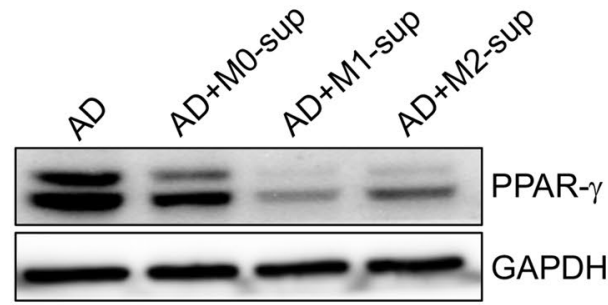

b
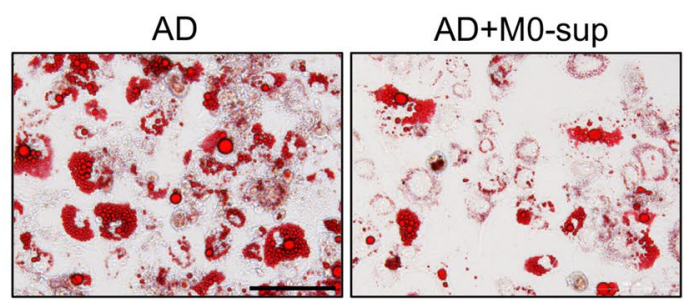

AD+M1-sup

AD+M2-sup
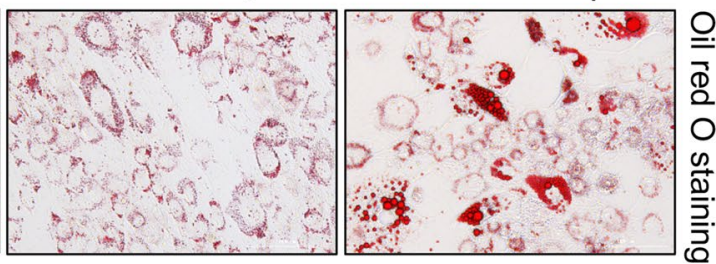

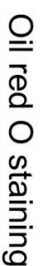

d
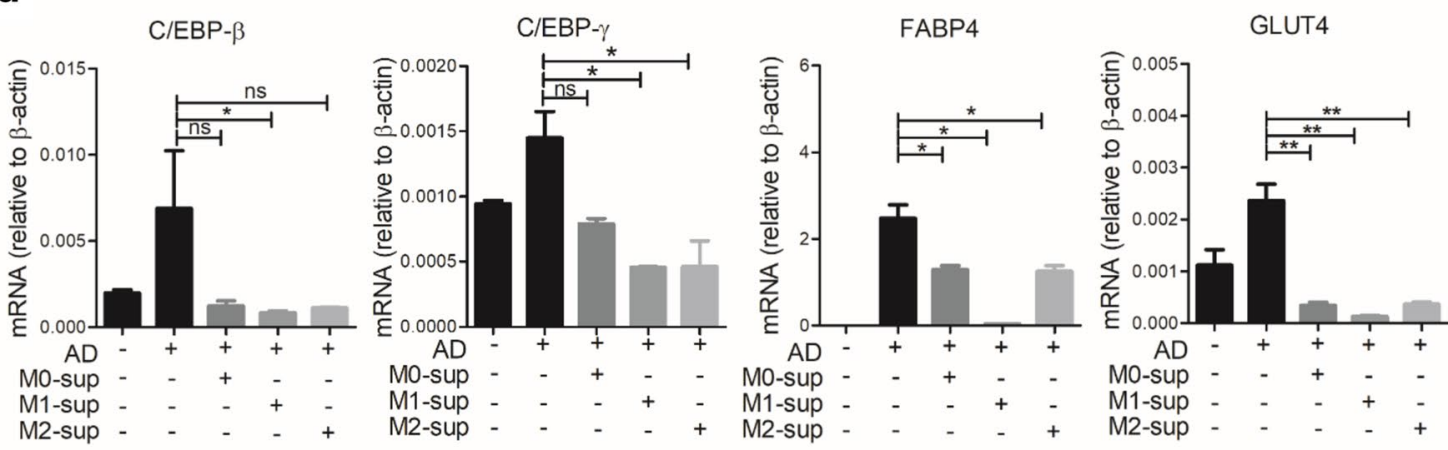

\section{e}

\section{Pre-treatment}
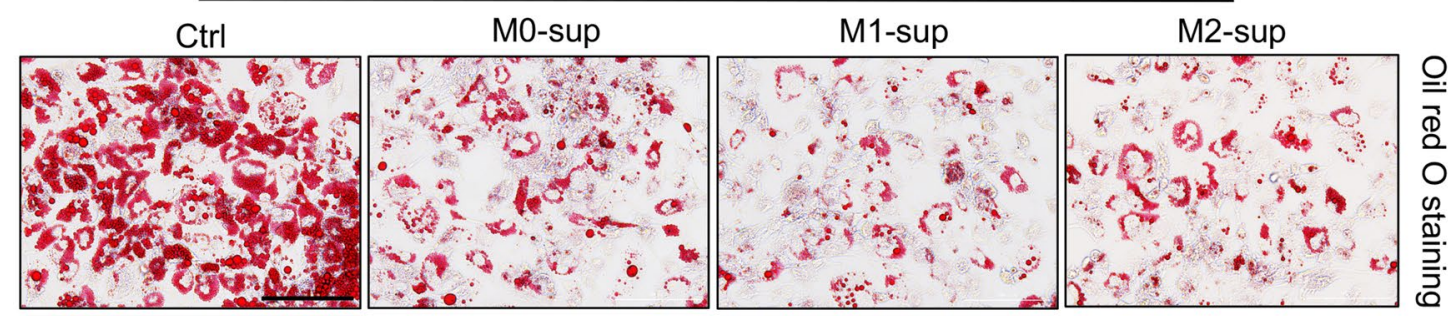

$A D$

Fig. 1 Macrophage-derived supernatants inhibit adipocyte differentiation of hADSCs. a Scheme of the experimental procedure. hADSCs $\left(2 \times 10^{4}\right)$ were plated and cultured with adipogenesis-inducing medium (AD) with or without the supernatants from different macrophage subtypes at the ratio of 1:1 in 48-well plate, medium was changed every 3 days. $\mathbf{b}$ hADSCs cells that were induced to undergo adipogenic differentiation were fixed for triglycerides staining with Oil Red O to show lipid droplets. $\mathbf{c}$ Western blot analysis of the protein levels of PPAR- $\gamma$ in hADSCs cultured in different conditions for 5 days. $\mathbf{d}$ Expression of adipogenic genes were measured by Q-PCR assay on day 3. e hADSCs were pre-treated with the specific supernatant for $24 \mathrm{~h}$, then changed to adipogenic inducing medium without supernatant, lipid droplets in hADSCs were revealed by Oil Red $\mathrm{O}$ staining after 9 days. Scale bars are $100 \mu \mathrm{m}$ 


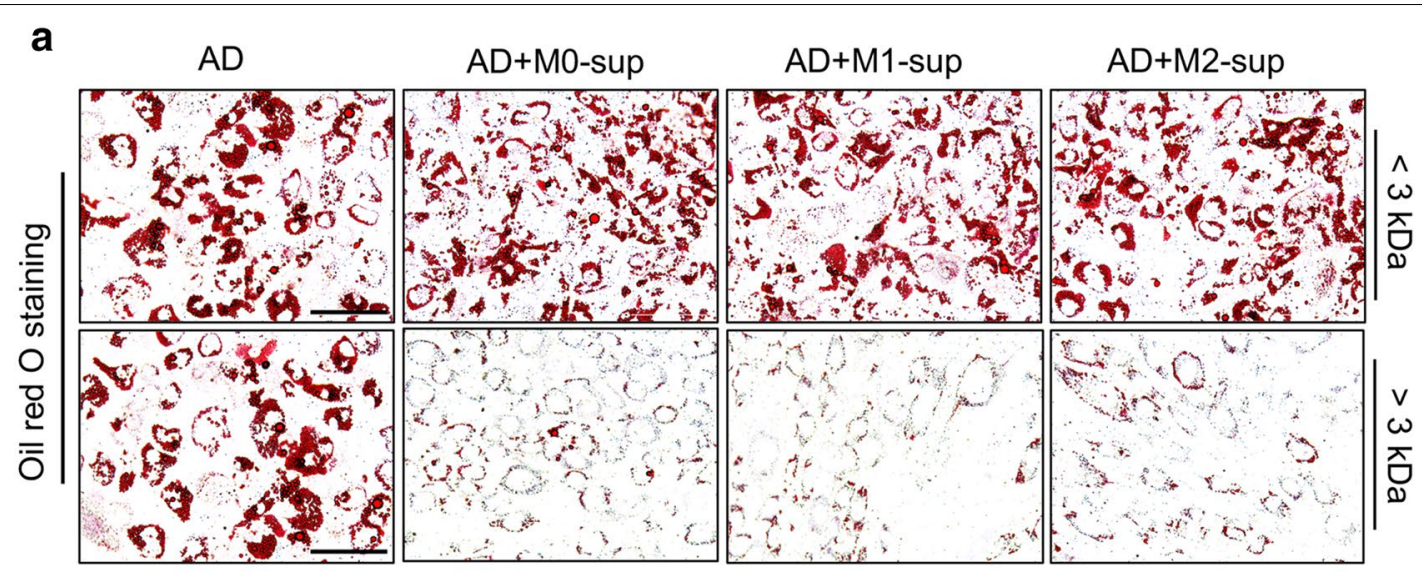

b

PPAR- $\gamma$

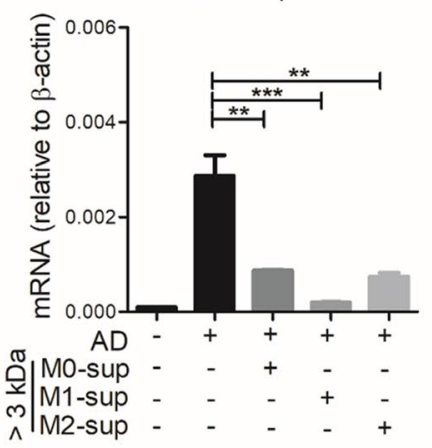

GLUT4

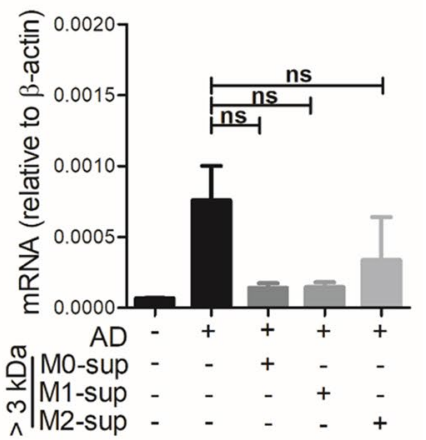

C/EBP- $\alpha$

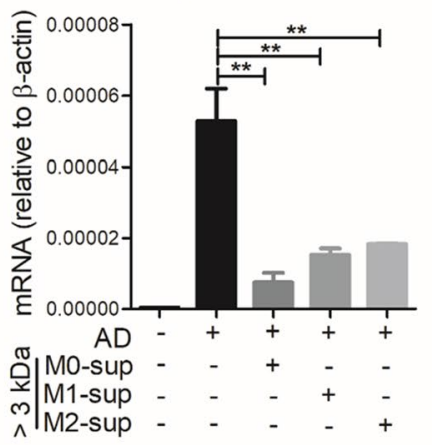

LPL

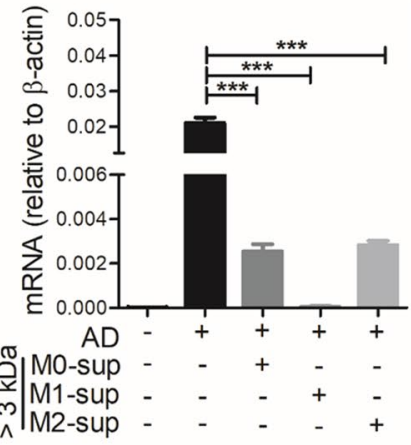

FABP4

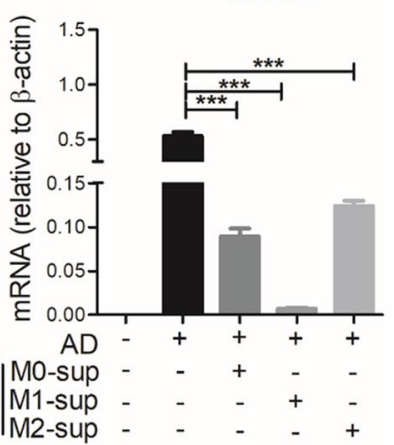

C

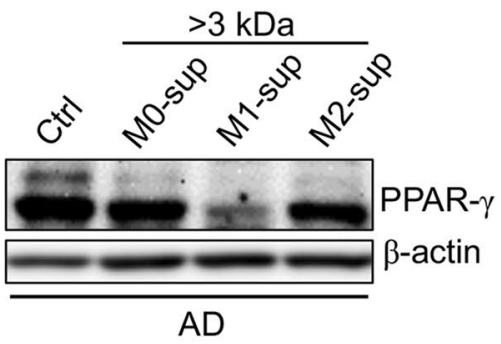

Fig. 2 The adipogenesis-inhibiting effect of macrophage supernatants is dependent on secretory macromolecule. a hADSCs were cultured with adipogenesis-inducing medium (AD) with or without the supernatant from different macrophage subtypes ( $>3 \mathrm{kDa}$ or $<3 \mathrm{kDa}$ ) at the ratio of 1:1 in 48 plate, medium was changed every 3 days. After being cultured for 9 days, cells were fixed for triglycerides staining with Oil Red $O$ to show lipid droplets. $\mathbf{b}$ Expression of adipogenesis-related genes in hADSCs treated with macrophages-derived supernatant ( $>3 \mathrm{kDa}$ ) was measured by Q-PCR on day 3. c Western blot analysis of the protein levels of PPAR-y in hADSCs cultured with different condition medium. All scale bars, $100 \mu \mathrm{m}$

(See figure on next page.)

Fig. 3 TNF- $\alpha$ and IL-1 $\beta$ repress the adipogenesis of hADSCs. a hADSCs were cultured with adipogenesis-inducing medium (AD) with or without IL-6, IL-12, TNF- $a$ and IL-1 $\beta$ ( $5 \mathrm{ng} / \mathrm{mL}$ each), respectively. Medium was changed every 3 days. After being cultured for 9 days, cells were fixed for triglycerides staining with Oil Red O. b Expression of adipogenesis-related genes in hADSCs treated with TNF-a/lL-1 $\beta$ (0.1 ng/mL each) was determined by Q-PCR assay on day 3. c Western blot analysis of PPAR- $\gamma$ in adipogenic hADSCs interfered with TNF-a or IL-1 $\beta$ cytokines at different concentration. Scale bars, $100 \mu \mathrm{m}$ 

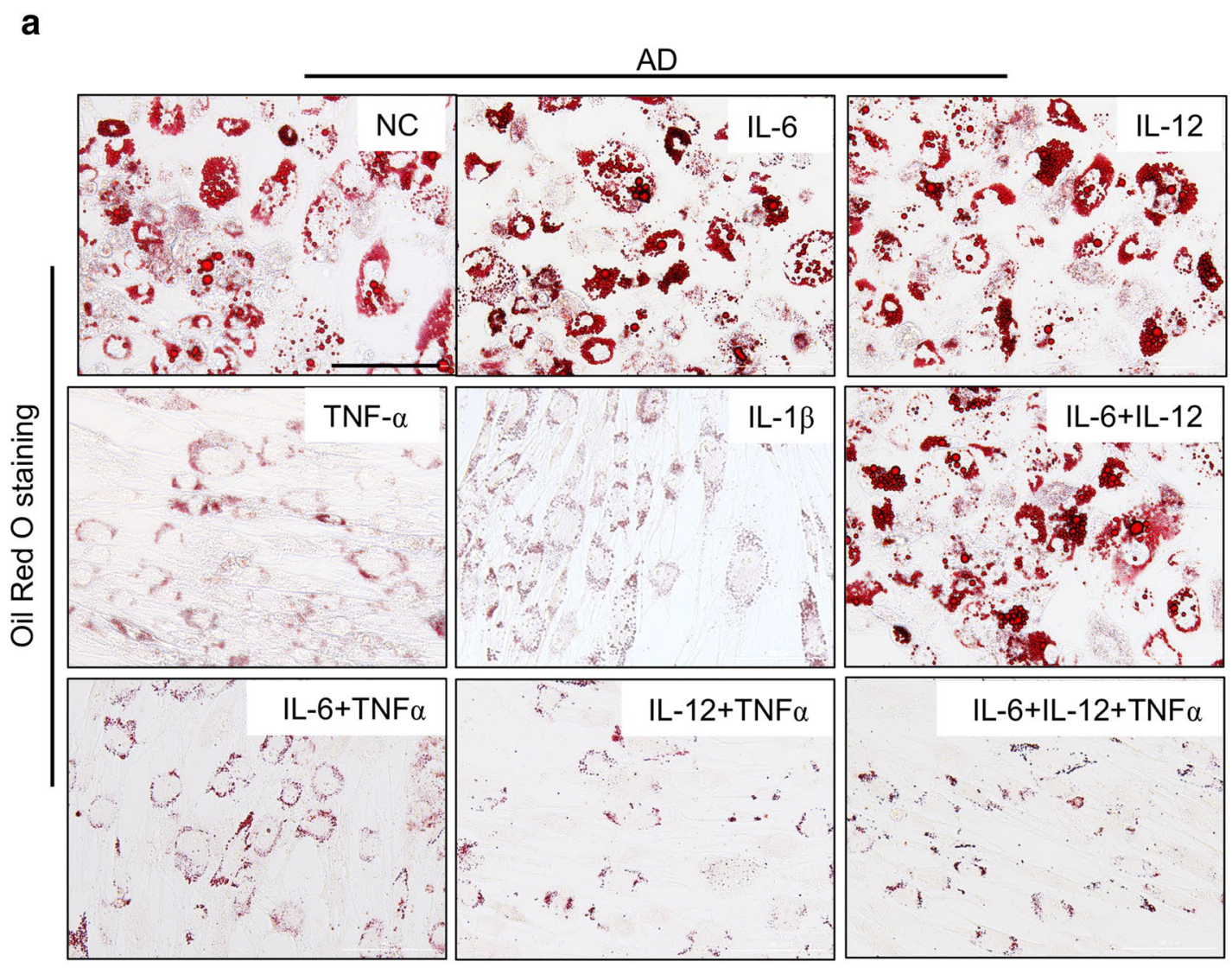

b

PPAR- $\gamma$

C/EBP- $\gamma$
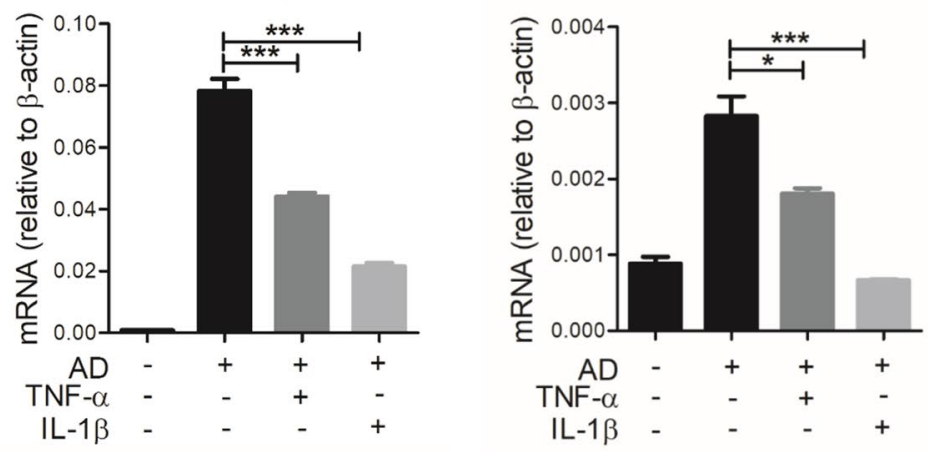

FABP4

GLUT4

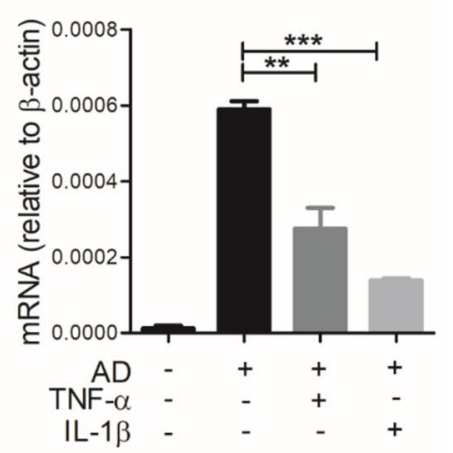

LPL
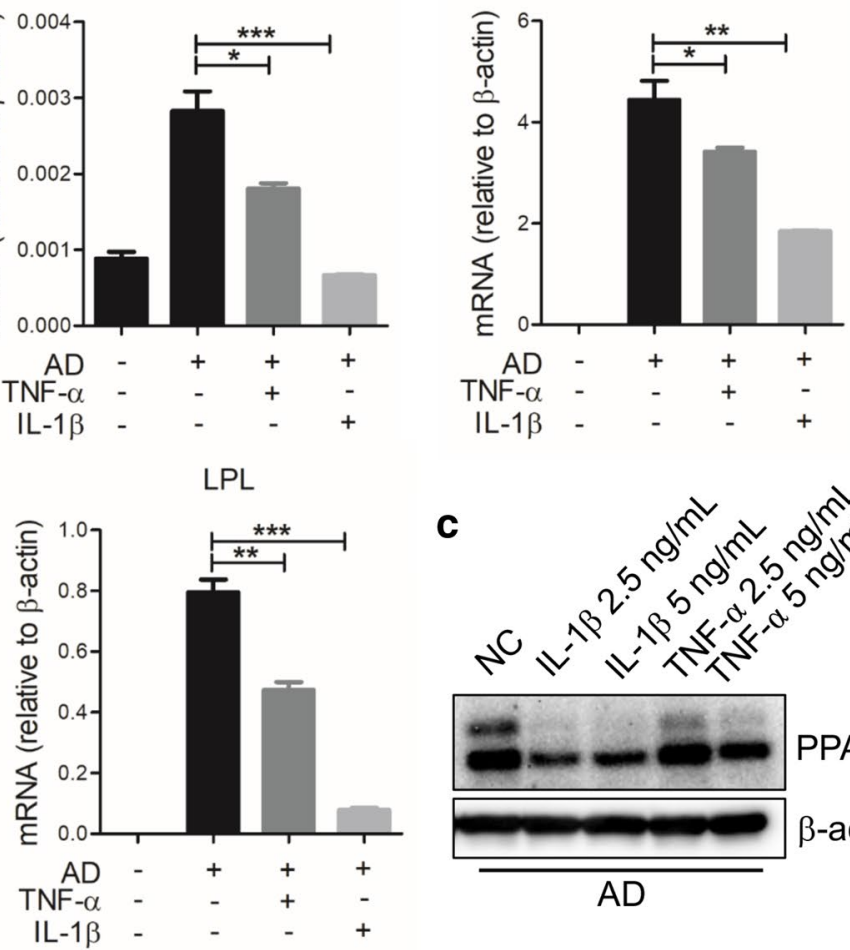

c

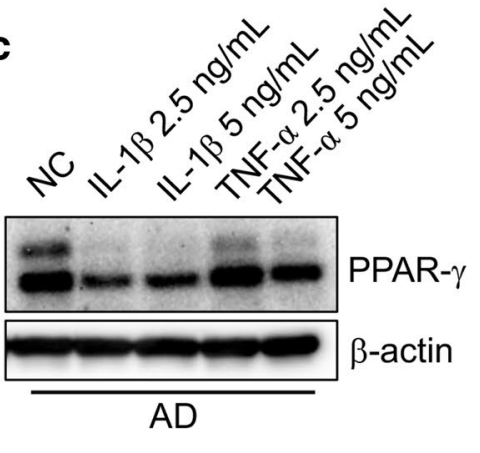




\section{TNF- $\alpha$ and IL-1 $\beta$ mediate the anti-adipogenic effect of M1-sup}

Given that pro-inflammatory cytokines TNF- $\alpha$ and IL-1 $\beta$ can inhibit adipogenic differentiation of hADSCs, we next investigated whether the two cytokines were responsible for the adipogenesis-inhibiting effect of M1-sup. We induced adipogenic differentiation in hADSCs in the presence of M1-sup as described above, but with specific antibodies targeting TNF- $\alpha$ and IL- $1 \beta$ being added. We found that neutralization of these cytokines abolished the anti-adipogenic effect of M1-sup, as significantly more lipid droplets were observed when compared with hADSCs treated only with the M1-sup (Fig. 4a). The repression of lipogenic genes, such as PPAR- $\gamma$, Glut4, FABP4 and $\mathrm{C} / \mathrm{EBP}-\alpha$, by $\mathrm{M} 1$-sup was also partially reversed with anti-TNF- $\alpha$ and anti-IL-1 $\beta$ blockage (Fig. $4 \mathrm{~b}, \mathrm{c}$ ). It is worth noting that the failure to fully abrogate the inhibition of M1-sup may result from an incomplete blockade

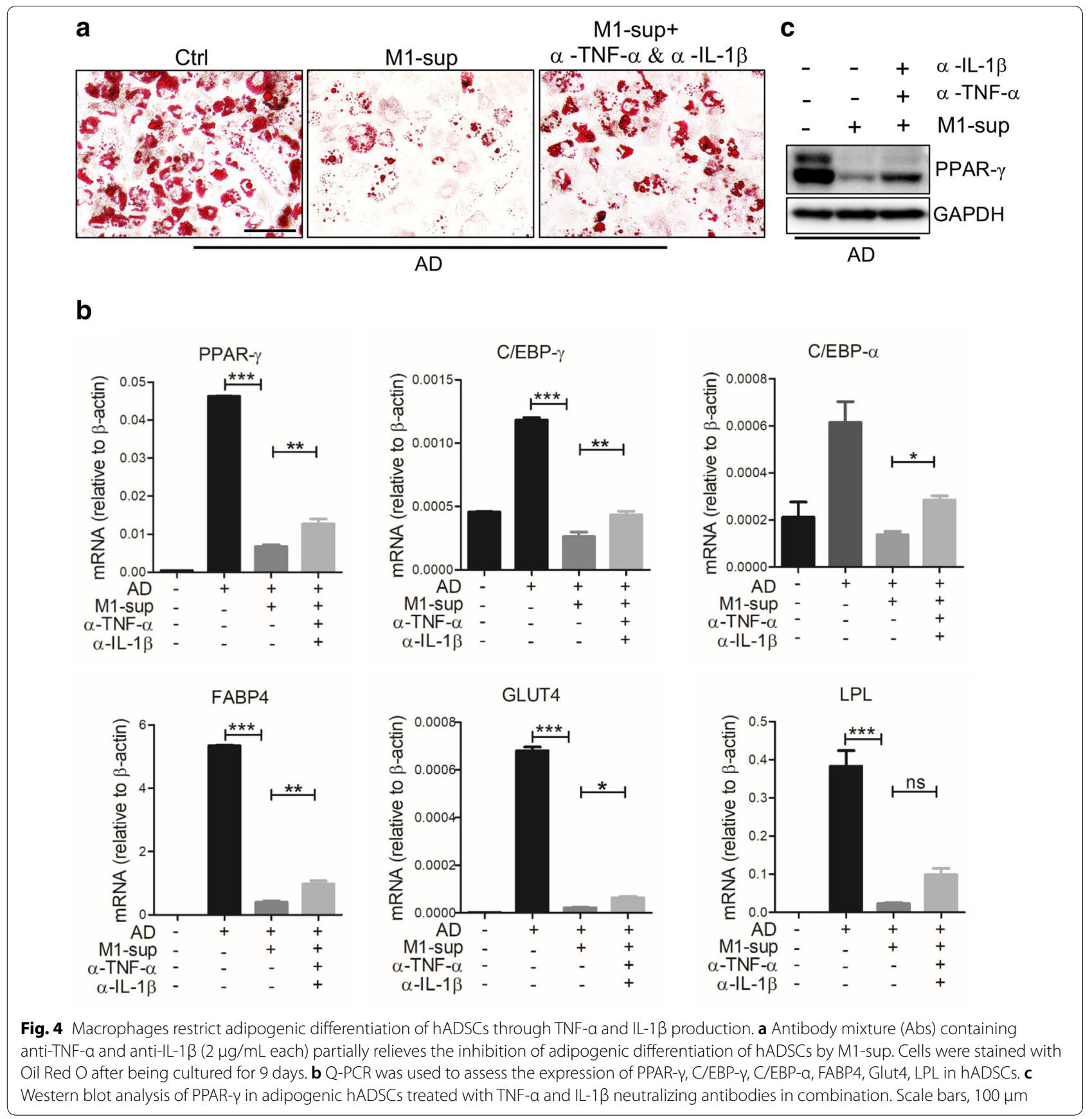


of those cytokines. Collectively, data above indicate that pro-inflammatory cytokines TNF- $\alpha / \mathrm{IL}-1 \beta$ were responsible for the inhibitory effect of M1-sup. Considering that there are other components in macrophage supernatant, we performed other experiments and found that exosomes secreted by M1 macrophage can also inhibit adipogenic differentiation of hADSCs, which will be reported in a separate study.

\section{Discussion}

Macrophages are now recognized as crucial regulators of physiological and pathological remodeling of adipose tissue. They can clear cellular debris and participate in tissue immune surveillance. Adipose tissue macrophages (ATMs) also function in lipid buffering [34]. Studies have shown that induction of brown adipogenesis with B3-adrenoceptor (ADRB3) agonist treatment is triggered by the recruitment of macrophages to vulnerable white adipocytes undergoing agonist-mediated cell death [35]. Recent work indicates that ATMs can clear adipose of dying adipocytes via arachidonate 15-lipoxygenase [36]. While macrophages play a critical role in regulating the adipose tissue homeostasis, how macrophages regulate adipogenesis remains to be fully addressed.
We tested the effects of M0, M1 or M2-polarized macrophages derived from THP1 monocytes on the lipogenic behavior of hADSCs in vitro. The results showed that they can all inhibit adipogenic differentiation, although the M1 macrophages are most potent. M1 macrophages were found to exert their inhibitory effect through secreting TNF- $\alpha$ and IL- $1 \beta$, but not IL-12 and IL-6. These in vitro results are in contrast to a report from Zhu et al. who demonstrated that ADSCs from obese pigs showed enhanced adipogenic propensity, which was abolished by anti-TNF- $\alpha$ antibody treatment, additionally, ADSCs from lean pigs showed enhanced adipogenesis when treated with TNF- $\alpha$ [37]. This difference may result from species difference or different ADSCs status originating in obese or lean individual. However, our finding is consistent with the report that TNF- $\alpha$ inhibits adpogenic differentiation of ADSCs [38]. It appears that how macrophages act on ADSCs under various physiological and pathological conditions still needs to be further explored.

Exosomes, a type of extracellular vesicles, play an important role in cellular communication as they are rich in mRNA, miRNA, and proteins that can regulate cellular signaling [39, 40]. Previous studies have report that exosomes secreted by adipose-tissue specific

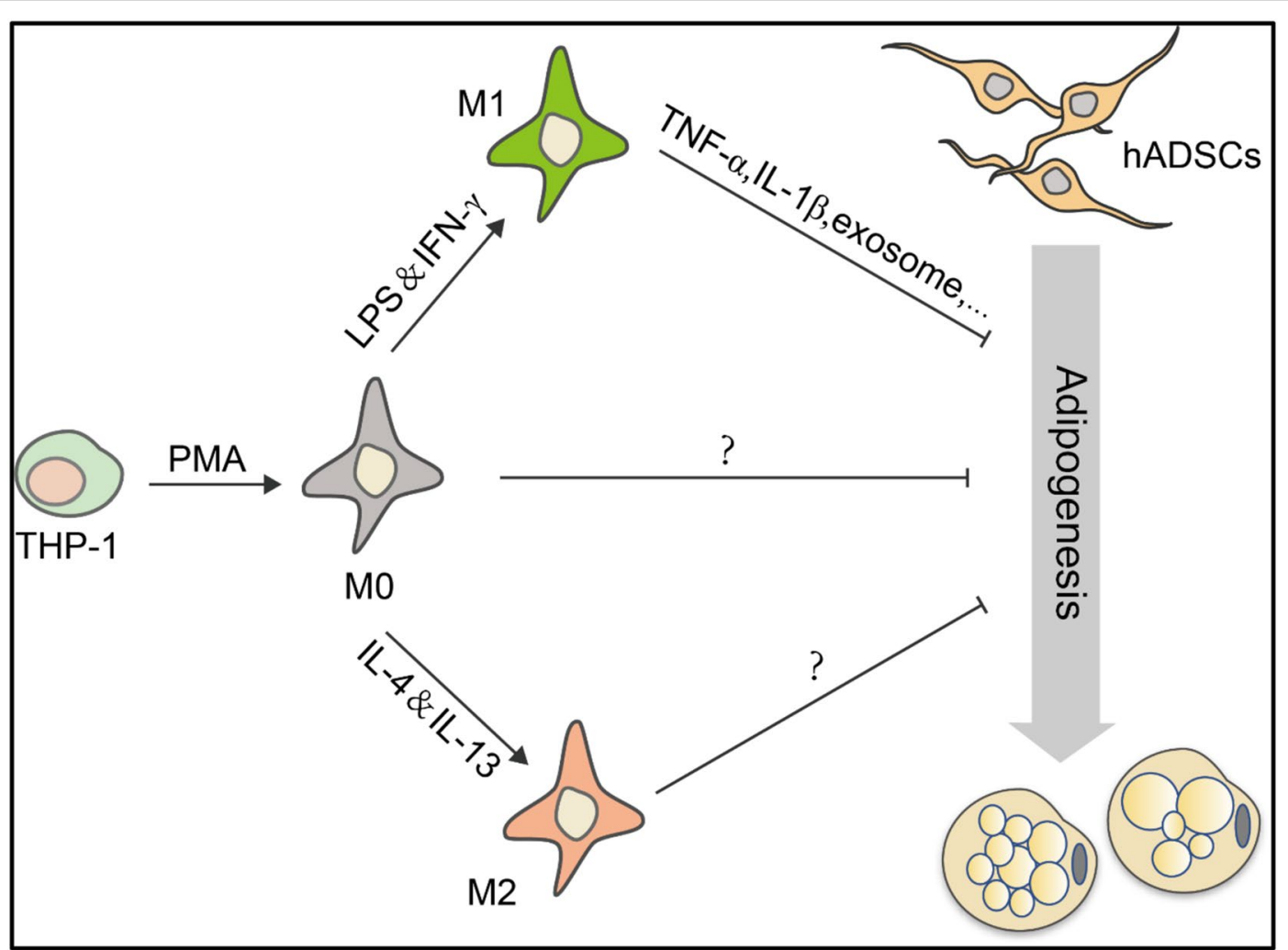

Fig. 5 A schematic diagram depicting the inhibitory effects of macrophages on adipocyte differentiation of hADSCs. M0, M1 and M2 macrophages can all inhibit adipogenic differentiation of hADSCS. M1 macrophage exert inhibitory effects by secreting inflammatory cytokines TNF-a and IL-1 $\beta$ 
macrophages in obese patients regulate the phosphorylation of AKT signaling pathway by inhibiting the expression of PPAR- $\gamma$ and Glut4, thus affect the insulin sensitivity of the body [41]. It is possible that exosomes derived from macrophages may also have an impact on adipogenic differentiation of hADSCs.

While we demonstrated an inhibitory effect of macrophages on the adipocyte differentiation of hADSC in vitro, whether the findings can be translated to the physiological and pathological conditions in vivo remains to be verified. Mouse models with the cytokine receptors being specifically deleted in ADSCs may help to confirm the role of inflammatory cytokines during adipogenesis. How different subsets of macrophages interact with ADSCs in vivo also needs to be delineated. Does the inhibition of adipocyte differentiation by cytokines have a beneficial effect? Is it possible that the suppression of adipocyte differentiation would endow the ADSCs an enhanced function in maintaining tissue homeostasis? It is well documented that MSCs exposed to inflammatory cytokines can acquire an increased immunomodulatory function [9]. A better understanding of the interaction between macrophages and hADSCs may help the clinical management of obesity and related diseases.

Taken together, we demonstrated that pro-inflammation cytokines derived from M1 macrophage inhibit the adipogenic differentiation of hADSCs (Fig. 5). Given that TNF- $\alpha / \mathrm{IL}-1 \beta$ can also endow MSCs immunosuppressive function, we speculate that there might be a counterbalance between adipogenesis potential and immunosuppressive function of MSCs, namely, the anti-adipogenic effect of pro-inflammatory cytokines may be evolutionary embedded to facilitate the immunosuppressive function of MSCs.

\section{Conclusion}

Our study demonstrated an inhibitory effect of macrophages on the adipogenic differentiation of hADSCs in vitro. While M0, M1 and M2 polarized macrophages can all inhibit adipocyte differentiation, M1 macrophages possess the most potent effect that is mediated by TNF- $\alpha$ and IL-1 $\beta$. Our findings expand the knowledge about the interaction between macrophages and hADSCs as well as the fate determination of hADSCs.

\section{Materials and methods \\ Cell line}

THP-1 cells, which were derived from the peripheral blood of a one-year-old boy with acute monocytic leukemia (American Type Culture Collection, ATCC), were cultured with RPMI-1640 medium (Hyclone) supplemented with $10 \%$ fetal bovine serum (FBS, Hyclone), 100
$\mathrm{U} / \mathrm{mL}$ penicillin and $100 \mathrm{~g} / \mathrm{mL}$ streptomycin (Invitrogen) in a humidified incubator under $5 \% \mathrm{CO}_{2}$ at $37^{\circ} \mathrm{C}$.

\section{Induction of THP-1 derived macrophages}

The macrophages were obtained by treating THP-1 cells with PMA (Sigma, USA) (100 ng/mL) for $48 \mathrm{~h}$. The matured macrophages were exposed to LPS $(100 \mathrm{ng} / \mathrm{mL})$ and IFN- $\gamma(20 \mathrm{ng} / \mathrm{mL})$ for $48 \mathrm{~h}$ to obtain M1 polarized macrophage (classical activated macrophage). M2-polarized macrophage (alternatively activated macrophage) were obtained by treatment with IL-4 and IL-13 (20 ng/ $\mathrm{mL}$ each) for $48 \mathrm{~h}$. All of the cytokines were from PeproTech, USA.

\section{Isolation and culture of hADSCs}

ADSCs were obtained from the adipose tissue of lipoaspirate samples following the protocols approved by the Ethics Committee of Soochow University. Informed consent was obtained from each patient. Fresh adipose tissues were washed with phosphate-buffered saline (PBS) in a few more times and then digested with collagenase II (Gibco, USA) for $60-90 \mathrm{~min}$ at $37^{\circ} \mathrm{C}$ in a shaker. After centrifuging at $500 \mathrm{~g}$ for $8 \mathrm{~min}$, the stromal vascular cell fraction suspension was cultured with DME/F12 medium (Hyclone, USA) containing 10\% FBS, $100 \mathrm{U} / \mathrm{mL}$ penicillin, $100 \mathrm{~g} / \mathrm{mL}$ streptomycin, $2 \mathrm{mM}$ glutamine and basic fibroblastic growth factor (bFGF) (complete medium) in specific T75 flask. The medium was changed every 3 days and cells were routinely passaged by trypsin digestion. hADSCs were characterized for surface markers by flow cytometer at 3th passage.

\section{Preparation of macrophage supernatants}

M0, M1 or M2 macrophages were obtained as described above. Cells were washed with PBS three times and cultured with fresh DMEM/High complete medium at $37{ }^{\circ} \mathrm{C}, 5 \% \mathrm{CO}_{2}$. Collected the cell culture medium after 2 days and centrifuged at $2000 \times g, 4{ }^{\circ} \mathrm{C}$ for $10 \mathrm{~min}$. Discarded precipitates and supernatants were then transferred to molecular weight cut-off filter basing $3 \mathrm{kDa}$ and centrifuged at $5000 \times g, 4{ }^{\circ} \mathrm{C}$ for $30 \mathrm{~min}$. Finally, fractions greater than $3 \mathrm{kDa}$ (the upper layer) and less than $3 \mathrm{kDa}$ (the bottom layer) were separately collected and stored at $-80^{\circ} \mathrm{C}$.

\section{Adipogenic and osteogenic differentiation of hADSCs}

For adipogenic differentiation, hADSCs $\left(2 \times 10^{4} /\right.$ well $)$ were seeded into 48-well plate with DME/F12 complete medium. When cells were grown to $80 \%$ confluence, medium was changed to adipogenic medium consisting of $0.5 \mathrm{mM}$ 3-isobutyl-1-methylxanthin (IBMX, Sigma, USA), and $60 \mu \mathrm{M}$ indomethacin (Sigma, USA), $100 \mathrm{nM}$ dexamethasone (Sigma, USA), $10 \mu \mathrm{g} /$ 
$\mathrm{mL}$ insulin (Sigma, USA). Then adipogenic medium was changed every 3 days. Osteogenic differentiation of hADSCs was induced according to established protocols with minor modification. hADSCs were cultured with DMEM/low medium containing $10 \mathrm{nM}$ dexamethasone, $100 \mu \mathrm{M}$ L-ascorbic acids (Sigma, USA), $10 \mathrm{nM}$ $\beta$-glycerophosphate (Sigma, USA), the medium was changed every 3 days during the osteogenesis-inducing process.

\section{Oil Red O staining}

hADSCs that were induced to undergo adiogenic differentiation were fixed on day 9 with $4 \%$ paraformaldehyde for 15-30 min at room temperature and washed with PBS twice, then cells were stained with Oil Red O (Bio-Connect, Holland) working solution for $15 \mathrm{~min}$ and washed by double distilled water for at least 3 times.

\section{RNA isolation and real-time quantitative polymerase chain reaction (Q-PCR)}

Total RNAs were extracted from cells by Trizol reagent (Thermo, USA) according to the manufacturer's instructions. About $1 \mu \mathrm{g}$ of total RNAs from each sample were reverse-transcribed into cDNA using the PrimeScript ${ }^{\mathrm{TM}}$ RT reagent Kit (TaKaRa, Japan). The mRNA expressions were quantitatively assessed by SYBR Green-based Q-PCR in an Applied Biosystems PRISM 7900HT Fast Real-time PCR System. The primers were synthesized by Suzhou GENEWIZ institute. RNA expression levels were compared after normalization with $\beta$-actin. The primer sequences were shown in Table 1.

\section{Western blot analysis}

The poly vinylidene fluoride (PVDF) membrane was blocked with $2 \%$ bovine serum albumin (BSA) solution or $5 \%$ nonfat dried milk and then incubated with primary antibodies overnight at $4{ }^{\circ} \mathrm{C}$. Membrane was washed with $1 \times$ tris-buffered saline and tween 20 (TBST) and incubated with either anti-rabbit or anti-mouse IgG horseradish peroxidase-conjugated secondary antibody and finally exposed using ChemiDoc XRS imaging system. Western blot data shown in figures were representative of more than three independent experiments.

\section{Flow cytometry analysis}

Cells were harvested by trypsin digestion and washed with PBS twice and then stained with fluorescein isothiocyanate-conjugated or phycoerythrin-conjugated (monoclonal) antibodies for $30 \mathrm{~min}$ at $4{ }^{\circ} \mathrm{C}$, after incubation, cells were washed twice and resuspended in $200 \mu \mathrm{L}$ PBS. Finally, cells were detected by flow cytometer (BECKMAN, USA). Data was analyzed using Flowjo software.
Table 1 List of primers used for quantitative polymerase chain reaction ( $\beta$-actin was used as an internal control)

\begin{tabular}{|c|c|}
\hline Gene & Primer sequences \\
\hline \multicolumn{2}{|l|}{ PPAR- $\gamma$} \\
\hline Forward & 5'-TACTGTCGGTTTCAGAAATGCC-3' \\
\hline Reverse & 5'-GTCAGCGGACTCTGGATTCAG-3' \\
\hline \multicolumn{2}{|l|}{ C/EBP-a } \\
\hline Forward & 5'-CGAAGAGACGGCCCTTGCTG-3' \\
\hline Reverse & 5'-GGGATACATCCTCAGGGCCACA-3' \\
\hline \multicolumn{2}{|l|}{ C/EBP- $\beta$} \\
\hline Forward & 5'-CTTCAGCCCGTACCTGGAG-3' \\
\hline Reverse & 5'-GGAGAGGAAGTCGTGGTGC-3' \\
\hline \multicolumn{2}{|l|}{$C / E B P-\gamma$} \\
\hline Forward & 5'-ACTCCAGGGGTGAACGGAAT-3' \\
\hline Reverse & 5'-CATGGGCGAACTCTTTTTGCT-3' \\
\hline \multicolumn{2}{|l|}{ Glut4 } \\
\hline Forward & 5'-TGGGCGGCATGATTTCCTC-3' \\
\hline Reverse & 5'-GCCAGGACATTGTTGACCAG-3' \\
\hline \multicolumn{2}{|l|}{ LPL } \\
\hline Forward & 5'-TCATTCCCGGAGTAGCAGAGT-3' \\
\hline Reverse & 5'-GGCCACAAGTTTTGGCACC-3' \\
\hline \multicolumn{2}{|l|}{ FABP4 } \\
\hline Forward & 5'-ACTGGGCCAGGAATTTGACG-3' \\
\hline Reverse & 5'-CTCGTGGAAGTGACGCCTT-3' \\
\hline \multicolumn{2}{|l|}{ IL-6 } \\
\hline Forward & 5'-CAGCCCTGAGAAAGGAGACATG-3' \\
\hline Reverse & 5'-GGTTGTTTTCTGCCAGTGCCT-3' \\
\hline \multicolumn{2}{|l|}{ IL-12 } \\
\hline Forward & 5'-GATGGCCCTGTGCCTTAGTA-3' \\
\hline Reverse & 5'-TCAAGGGAGGATTTTTTGTGG-3' \\
\hline \multicolumn{2}{|l|}{$\mid L-1 \beta$} \\
\hline Forward & 5'-GACCTGAGCACCTTCTTTCCCT-3' \\
\hline Reverse & 5'-CATCGTGCACATAAGCCTCGT-3' \\
\hline \multicolumn{2}{|l|}{ TNF-a } \\
\hline Forward & 5'-GACAAGCCTGTAGCCCATGTTG-3' \\
\hline Reverse & 5'-TGGTTATCTCTCAGCTCCACGC-3' \\
\hline \multicolumn{2}{|l|}{ TGM2 } \\
\hline Forward & 5'-CAAGGCCCGTTTTCCACTAAG-3' \\
\hline Reverse & 5'-GAGGCGATACAGGCCGATG-3' \\
\hline \multicolumn{2}{|l|}{ CCL22 } \\
\hline Forward & 5'-ATCGCCTACAGACTGCACTC-3' \\
\hline Reverse & $5^{\prime}$-GACGGTAACGGACGTAATCAC-3' \\
\hline \multicolumn{2}{|l|}{$\beta$-actin } \\
\hline Forward & $5^{\prime}$-TTGCCGACAGGATGCAGAAGGA-3' \\
\hline Reverse & 5'-AGGTGGACAGCGAGGCCAGGAT-3' \\
\hline
\end{tabular}




\section{Quantification and statistical analysis}

Results were expressed as mean \pm SEM. The difference between various treatments were evaluated by either the two tailed Student's test or one-way ANOVA with Bonferroni post-test. Data analyses were performed using Graph Pad Prism software version 5.0. A value of $p<0.05$ was considered statistically significant.

\section{Supplementary information}

Supplementary information accompanies this paper at https://doi. org/10.1186/s13578-020-00450-y.

Additional file 1. Additional figures.

\section{Abbreviations}

hADSCs: Human adipose derived mesenchymal stem/stromal cells; TNF-a: Tumor necrosis factor $\alpha$; IFN- $\gamma$ : Interferon- $\gamma$; C/EBP: CCAAT/enhancer-binding protein CCAAT; PPAR- $\gamma$ : Peroxisome proliferator activated receptor $\gamma$; FABP-4: Fatty acid binding protein-4; GLUT4: Glucose transporter 4; LPL: Lipoprotein lipase; PMA: Phorbol-12-myristate-13-acetate; IL: Interleukin; TGM2: Transglutaminase 2; CCL22: CC chemokine ligand 22.

\section{Acknowledgements}

Not applicable.

\section{Authors' contributions}

$\mathrm{HM}, \mathrm{YL}, \mathrm{CS}$ and $\mathrm{YS}$ contributed to study design. $\mathrm{HM}, \mathrm{YL}, \mathrm{LS}, \mathrm{RL}, \mathrm{XL}, \mathrm{QS}$ and $\mathrm{YW}$ performed the experiment and contributed to the data collection, analysis and interpretation. HM, YL and CS were involved in the writing of the manuscript. All authors read and approved the final manuscript.

\section{Funding}

This study was supported by grants from the National Key R\&D Program of China (2018YFA0107500), National Natural Science Foundation of China (31961133024, 81530043 and 81930085), the Scientific Innovation Project of the Chinese Academy of Science (XDA16020403, XDA16010403), and the State Key Laboratory of Radiation Medicine and Protection, Soochow University (GZN1201804 and GZN1201903).

\section{Availability of data and materials}

The datasets used and/or analyzed during the current study are available from the corresponding author on reasonable request

\section{Ethics approval and consent to participate}

Lipoaspirate samples were obtained following the protocol approved by the Ethics Committee of Soochow University. Informed consent was obtained from each patient.

\section{Consent for publication}

All authors read and approved the paper.

\section{Competing interests}

The authors declare that they have no competing interests.

\section{Author details}

${ }^{1}$ The First Affiliated Hospital of Soochow University, Institutes for Translational Medicine, State Key Laboratory of Radiation Medicine and Protection, Key Laboratory of Stem Cells and Medical Biomaterials of Jiangsu Province, Medical College of Soochow University, Suzhou 215123, Jiangsu, China. ${ }^{2}$ CAS Key Laboratory of Tissue Microenvironment and Tumor, Shanghai Institute of Nutrition and Health, Shanghai Institutes for Biological Sciences, University of Chinese Academy of Sciences, Chinese Academy of Sciences, Shanghai 200031, China. ${ }^{3}$ Institutes for Translational Medicine, State Key Laboratory of Radiation Medicine and Protection, Soochow University, Suzhou 215123, Jiangsu, China.
Received: 1 April 2020 Accepted: 11 July 2020

Published online: 20 July 2020

\section{References}

1. Friedenstein AJ, Chailakhyan RK, Latsinik NV, et al. Stromal cells responsible for transferring the microenvironment of the hemopoietic tissues Cloning in vitro and retransplantation in vivo. Transplantation. 1974;17(4):331-40.

2. Barry FP, Murphy JM. Mesenchymal stem cells: clinical applications and biological characterization. Int J Biochem Cell Biol. 2004;36(4):568-84.

3. Kassem M, Kristiansen M, Abdallah BM. Mesenchymal stem cells: cell biology and potential use in therapy. Basic Clin Pharmacol Toxicol. 2004;95(5):209-14.

4. Pittenger MF, Mackay AM, Beck SC, et al. Multilineage potential of adult human mesenchymal stem cells. Science. 1999;284(5411):143-7.

5. Jiang $Y$, Jahagirdar BN, Reinhardt RL, et al. Pluripotency of mesenchymal stem cells derived from adult marrow. Nature. 2002;418(6893):41-9.

6. Uccelli A, Moretta L, Pistoia V. Mesenchymal stem cells in health and disease. Nat Rev Immunol. 2008;8(9):726-36.

7. Lowe CE, Orahilly S, Rochford JJ. Adipogenesis at a glance. J Cell Sci. 2011;124(Pt 16):2681-6.

8. Matsushita K, Dzau VJ. Mesenchymal stem cells in obesity: insights for translational applications. Lab Invest. 2017;97(10):1158-66.

9. Shi Y, Wang Y, Li Q, et al. Immunoregulatory mechanisms of mesenchymal stem and stromal cells in inflammatory diseases. Nat Rev Nephrol. 2018;14(8):493-507.

10. Ren G, Su J, Zhang L, et al. Species variation in the mechanisms of mesenchymal stem cell-mediated immunosuppression. Stem Cells. 2009;27(8):1954-62.

11. Su J, Chen X, Huang Y, et al. Phylogenetic distinction of iNOS and IDO function in mesenchymal stem cell-mediated immunosuppression in mammalian species. Cell Death Differ. 2014;21(3):388-96.

12. Lim JY, Ryu DB, Lee SE, et al. Mesenchymal Stem Cells (MSCs) Attenuate Cutaneous Sclerodermatous Graft-Versus-Host Disease (Scl-GVHD) through Inhibition of Immune Cell Infiltration in a Mouse Model. J Invest Dermatol. 2017;137(9):1895-904.

13. Chen C, Liang J, Yao G, et al. Mesenchymal stem cells upregulate Treg cells via sHLA-G in SLE patients. Int Immunopharmacol. 2017:44:234-41.

14. Shu J, He X, Li H, et al. The Beneficial Effect of Human Amnion Mesenchymal Cells in Inhibition of Inflammation and Induction of Neuronal Repair in EAE Mice. J Immunol Res. 2018;2018:5083797.

15. Kim SY, Nair MG. Macrophages in wound healing: activation and plasticity. Immunol Cell Biol. 2019;97(3):258-67.

16. Murray PJ. Macrophage polarization. Annu Rev Physiol. 2017;79:541-66.

17. Biswas SK, Mantovani A. Macrophage plasticity and interaction with lymphocyte subsets: cancer as a paradigm. Nat Immunol. 2010;11(10):889-96.

18. Olefsky JM, Glass CK. Macrophages, inflammation, and insulin resistance. Annu Rev Physiol. 2010;72:219-46.

19. Weisberg SP, Mccann D, Desai M, et al. Obesity is associated with macrophage accumulation in adipose tissue. J Clin Invest. 2003;112(12):1796-808

20. Kerru N, Gummidi L, Bhaskaruni S, et al. A comparison between observed and DFT calculations on structure of 5-(4-chlorophenyl)-2-amino-1,3,4thiadiazole. Sci Rep. 2019;9(1):19280.

21. Rendra E, Riabov V, Mossel DM, et al. Reactive oxygen species (ROS) in macrophage activation and function in diabetes. Immunobiology. 2019;224(2):242-53.

22. Du L, Lin L, Li Q, et al. IGF-2 preprograms maturing macrophages to acquire oxidative phosphorylation-dependent anti-inflammatory properties. Cell Metab. 2019;29(6):1363-75.

23. Rosen ED, Spiegelman BM. What we talk about when we talk about fat. Cell. 2014;156(1-2):20-44.

24. Zheng C, Yang Q, Cao J, et al. Local proliferation initiates macrophage accumulation in adipose tissue during obesity. Cell Death Dis. 2016;7:e2167.

25. Zheng C, Yang Q, Xu C, et al. CD11b regulates obesity-induced insulin resistance via limiting alternative activation and proliferation of adipose tissue macrophages. Proc Natl Acad Sci USA. 2015;112(52):E7239-48. 
26. Cho DI, Kim MR, Jeong HY, et al. Mesenchymal stem cells reciprocally regulate the M1/M2 balance in mouse bone marrow-derived macrophages. Exp Mol Med. 2014;46:e70.

27. Philipp D, Suhr L, Wahlers T, et al. Preconditioning of bone marrowderived mesenchymal stem cells highly strengthens their potential to promote IL-6-dependent M2b polarization. Stem Cell Res Ther. 2018;9(1):286

28. Vasandan AB, Jahnavi S, Shashank C, et al. Human Mesenchymal stem cells program macrophage plasticity by altering their metabolic status via a PGE2-dependent mechanism. Sci Rep. 2016;6:38308.

29. Zhao H, Shang Q, Pan Z, et al. Exosomes from adipose-derived stem cells attenuate adipose inflammation and obesity through polarizing M2 macrophages and beiging in white adipose tissue. Diabetes. 2018;67(2):235-47.

30. Zhang Y, Bose T, Unger RE, et al. Macrophage type modulates osteogenic differentiation of adipose tissue MSCs. Cell Tissue Res. 2017;369(2):273-86.

31. Chanput W, Mes JJ, Wichers HJ. THP-1 cell line: an in vitro cell model for immune modulation approach. Int Immunopharmacol. 2014;23(1):37-45.

32. Bonora M, Wieckowsk MR, Chinopoulos C, et al. Molecular mechanisms of cell death: central implication of ATP synthase in mitochondrial permeability transition. Oncogene. 2015;34(12):1608.

33. Uysal KT, Wiesbrock SM, Marino MW, et al. Protection from obesityinduced insulin resistance in mice lacking TNF-alpha function. Nature. 1997;389(6651):610-4.

34. Boutens L, Stienstra R. Adipose tissue macrophages: going off track during obesity. Diabetologia. 2016;59(5):879-94
35. Lee YH, Kim SN, Kwon HJ, et al. Adipogenic role of alternatively activated macrophages in beta-adrenergic remodeling of white adipose tissue. Am J Physiol Regul Integr Comp Physiol. 2016;310(1):R55-65.

36. Kwon HJ, Kim SN, Kim YA, et al. The contribution of arachidonate 15-lipoxygenase in tissue macrophages to adipose tissue remodeling. Cell Death Dis. 2016;7(6):e2285.

37. Zhu XY, Ma S, Eirin A, et al. Functional plasticity of adipose-derived stromal cells during development of obesity. Stem Cells Transl Med. 2016;5(7):893-900.

38. Liu S, Yang Y, Wu J. TNFalpha-induced up-regulation of miR-155 inhibits adipogenesis by down-regulating early adipogenic transcription factors. Biochem Biophys Res Commun. 2011;414(3):618-24.

39. Denzer K, Kleijmeer MJ, Heijnen HF, et al. Exosome: from internal vesicle of the multivesicular body to intercellular signaling device. J Cell Sci. 2000;113(Pt 19):3365-74.

40. Qin J, Xu Q. Functions and application of exosomes. Acta Pol Pharm. 2014;71(4):537-43.

41. Ying W, Riopel M, Bandyopadhyay G, et al. Adipose tissue macrophagederived exosomal miRNAs can modulate in vivo and in vitro insulin sensitivity. Cell. 2017;171(2):372-84.

\section{Publisher's Note}

Springer Nature remains neutral with regard to jurisdictional claims in published maps and institutional affiliations.
Ready to submit your research? Choose BMC and benefit from:

- fast, convenient online submission

- thorough peer review by experienced researchers in your field

- rapid publication on acceptance

- support for research data, including large and complex data types

- gold Open Access which fosters wider collaboration and increased citations

- maximum visibility for your research: over 100M website views per year

At BMC, research is always in progress.

Learn more biomedcentral.com/submissions 